\title{
Precise Information of Hypermetropia
}

\author{
Partha Haradhan Chowdhury ${ }^{*}$ and Brinda Haren Shah ${ }^{2}$ \\ 1M.Optom, Associate Professor, Principal, Department of Optometry, Shree Satchandi \\ Jankalyan Samiti Netra Prasikshan Sansthan, Pauri, Affiliated to Uttarakhand State \\ Medical Faculty, Dehradun, India
}

${ }^{2}$ M.Optom, Guest Lecturer, Department of Optometry, Shree Satchandi Jankalyan

\section{Short Communication}

Volume 3 Special Issue 2

Received Date: October 02, 2018

Published Date: October 22, 2018

Samiti Netra Prasikshan Sansthan, Pauri, Affiliated to Uttarakhand State Medical Faculty, Dehradun, India

*Corresponding author: M. Optom, Associate Professor, Principal, Department of Optometry, Shree Satchandi Jankalyan Samiti Netra Prasikshan Sansthan, Pauri, Affiliated to Uttarakhand State Medical Faculty, Dehradun, India, Email: optometrypublish@gmail.com

\section{Abstract}

This paper describes about Hypermetropia, its grades, associations and treatment methodology.

Keywords: Hypermetropia; Cyclodemia

\section{Introduction}

It is conditions where parallel rays of light are coming from the infinity are brought to focus behind the retina when accommodation is at rest.

In case of hypermetropic refractive error, patient will usually complain of headache as accommodation is being stimulated. Because, in presence of hypermetropia, the light is focused behind the retina, and when patient will try to focus that image on the retina by applying accommodation, headache is being created.

Most of the time, hypermetropia is associated with posterior shortening of the eyeball. Other reasons are: flattening of the cornea, low refractive index of the crystalline lens, displacement of the crystalline lens towards retina.

In case of Hypermetropia, there are also chances of developing glaucoma. Here, antero posterior length shortens in hypermetropia, but diameter of the crystalline lens is normal so angle of anterior chamber becomes shallow.
Hypermetropia is more dangerous compared to Myopia. In case of Hypermetropia, there are more chances of developing amblyopia as compared to Myopia. Hypermetropia is most commonly associated with Eso deviation due to over accommodation [1-3].

Most of the time Myopia is associated with Exodeviation. Compared to Eso deviation, magnitude of the prism power to treat Exo deviation is much more. In case of Myopia, accommodation is in rest condition, and there are less chances of over accommodation. Exo deviation is associated with intermittent stage and Eso deviation is in fixed stage. By this we can conclude that Hypermetropia is more dangerous as compared to Myopia.

At birth, mostly eye is in the hypermetropic stage and it is around 2.50 to $3.00 \mathrm{D}$. With increase in age, it is resolved due to eyeball growth. When anterior posterior eyeball is changed up to $1 \mathrm{~mm}$ then refractive error will be changed up to 3.00 D. Sometimes anterior posterior eyeball growth a change is associated with pathological. So, hypermetropia is very crucial compared to other refractive error. 


\section{Open Access Journal of Ophthalmology}

In hypermetropia, anterior posterior diameter becomes shorten. During ophthalmosocopy, optic disc shines and looks like optic neuritis. This is called pseudo optic nerve papillitis. Associated retina will also shine and is called shot silk retina. Very rare, but some hypermetropias are associated with asymmetrical face or one side least perfect development.

\section{Grades of Hypermetropia are as Follows:}

Low: $+0.25 \mathrm{D}-+3.00 \mathrm{D}$

Medium: +3.00 D - +5.00 D

High: > +5.00 D

Some amount of hypermetropia is compensated by implication of accommodation and contraction of ciliary muscle. Some amount of hypermetropia is revealed by the cycloplegic drugs. That exact amount of hypermetropia is called Latent Hypermetropia. The remaining portion which is in normal situation is uncorrected and termed as Manifest Hypermetropia. So, total hypermetropia is latent hypermetropia + manifest hyperemtropia.

Eg. Suppose patient have +0.75 Ds Hypermetropia, but by the superimpose of the ciliary muscle made by the patient, patient can read that letter. There will be complaining of headache. This type of Hypermetropia is called Facultative Hypermetropia. If the refractive error is large in amount, at that time patient is not able to compensate that amount of refractive error. This amount of uncorrected refractive error is called Absolute Hypermetropia. Suppose, hypermetropia is up to +5.00 Ds, patient is not able to compensate the amount. i.e. patient is able to compensate up to +1.00 Ds. So, remaining and total amount is absolute hypermetropia.

\section{Treatment}

Usually, hypermetropia is treated with cycloplegics or cyclodemia. Cyclodemia is a procedure where fogging should be done. Retinoscopy is performed and whatever neutralization power is obtained, fogging should be started with that neutralization power only. Eg. Suppose in case of Hypermetropia, neutralization is achieved at $+5.00 \mathrm{Ds}$, the starting point of the fogging lens is +5.00 Ds lens. For pediatric cases, cycloplegic refraction is very essential because latent amount of hypermetropia is suspected. After giving the full refractive correction, patient can be directed to spectacles, contact lenses or refractive surgery.

In case of spectacles, aspheric designs should be advised. In case of associated Eso deviation, full hypermetropic correction should be prescribed and in case of Exo deviation, full hypermetropic correction should not be prescribed.

\section{References}

1. William J Benjamin (2006) Borish's Clinical Refraction. 2nd (Edn.).

2. Theodore Grosvenor, Theodore P Grosvenor (2007) Primary Care Optometry. $5^{\text {th }}$ (Edn.).

3. Sir Stewart Duke-Elder, David Abrams (1978) DukeElder's Practice of refraction.

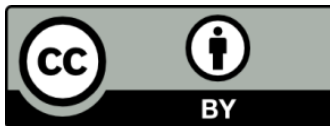

Partha Haradhan Chowdhury and Brinda Haren Shah. Precise Information of Hypermetropia. J Ophthalmol 2018, 3(S2): 000S2-018. 